\title{
Revisión
}

\section{Enfermedad por neumococo: desafíos actuales en los pacientes con enfermedad renal crónica y diabetes mellitus}

\author{
Jorge-Eduardo Rico-Fontalvo (D) ${ }^{1}$, Rodrigo Daza-Arnedo (D) ${ }^{2}$, Nehomar \\ Pájaro-Galvis (D) ${ }^{3} \square$, Víctor Leal-Martínez (D) ${ }^{4}$, Christian Pérez-Calvo (D) ${ }^{5}$, \\ Emilio Abuabara-Franco ${ }^{(D)}{ }^{3}$, Isabella Uparella-Gulfo ${ }^{[D}{ }^{3}$, Yasir \\ Valle-Villadiego ${ }^{(\mathbb{D}}{ }^{6}$, Laura Aguilera-Rios ${ }^{[D}{ }^{6}$, Carol Ceballos-Díaz ${ }^{[D} 7$, Jesús \\ Díaz-Gzmán ${ }^{(D)}{ }^{8}$ y Isabell Guete-García (iD) 9 \\ ${ }^{1}$ Comité de Nefrodiabetes, Asociación Colombiana de Nefrología e Hipertensión Arterial, Medellín, Colombia. \\ ${ }^{2}$ Comité de Nefrodiabetes, Asociación Colombiana de Nefrología e Hipertensión Arterial, Cartagena, Colombia. \\ ${ }^{3}$ Departamento de Medicina Interna, Facultad de Ciencias de la Salud, Universidad del Sinú, Cartagena. \\ ${ }^{4}$ Departamento de Medicina Interna, Clínica Somer, Rionegro, Colombia. \\ ${ }^{5}$ Departamento de Medicina Interna, Facultad de Ciencias de la Salud Universidad Libre, Barranquilla. \\ ${ }^{6}$ Servicio de Urgencias, Fundación Hospital San Carlos, Bogotá D.C., Colombia. \\ ${ }^{7}$ Facultad de Medicina, Universidad de Cartagena, Cartagena, Colombia. \\ ${ }^{8}$ Servicio de Urgencias, Hospital San Juan, Sahagún, Córdoba, Colombia. \\ ${ }^{9}$ Servicio de Urgencias, Clínica Blas de Lezo, Cartagena, Colombia.
}

Cómo citar: Rico-Fontalvo JE, Daza-Arnedo R, Pájaro-Galvis N, Leal-Martínez V, Pérez-Calvo C, Abuabara-Franco. Enfermedad por neumococo: desafíos actuales en los pacientes con enfermedad renal crónica y diabetes mellitus. Rev. Colomb. Nefrol. 2021 8(3), e510. https:/doi.org/ 10.22265/acnef.8.3.510

Recibido:

20/Ago/2020

Aceptado:

$14 / \mathrm{Feb} / 2021$

Publicado:

18/Ago/2021

\section{Resumen}

La enfermedad por neumococo se define como cualquier infección causada por la bacteria Streptococcus pneumoniae; esta se denomina invasiva cuando el aislamiento de este microorganismo se confirma en sitios corporales que son normalmente estériles, tales como la sangre y el líquido cefalorraquídeo, y no invasiva cuando la infección se produce en sitio corporales que normalmente no son estériles. La enfermedad renal crónica y la diabetes mellitus son factores de riesgo importantes para el desarrollo de enfermedad neumocócica debido a su estado de inmunosupresión, por lo que la vacunación antineumocócica es una medida preventiva fundamental en los pacientes que padecen estas condiciones.

Palabras clave: enfermedad renal crónica; diabetes mellitus; infecciones estreptocócicas.

$\checkmark$ Correspondencia: Transversal 54 41-117, Cartagena, Colombua. neho94@hotmail.com 


\title{
Pneumococal disease: current challenges in patients with chronic kidney disease and diabetes mellitus
}

\begin{abstract}
Pneumococcal disease is defined as any infection caused by the bacteria Streptococcus pneumoniae; it is called invasive when the isolation of this microorganism is confirmed in sites of the body that are normally sterile, such as blood and cerebrospinal fluid, and non-invasive when the infection occurs in sites of the body that are normally non-sterile. Chronic kidney disease and diabetes mellitus are important risk factors for the development of pneumococcal disease due to their status of immunosuppression; therefore, anti-pneumococcal vaccination is a fundamental preventive measure in patients who suffer from these conditions.
\end{abstract}

Keywords: chronic kidney disease; mellitus diabetes; streptococcal infections.

\section{Enfermedad neumocócica}

La enfermedad por neumococo (EPN), también conocida como neumocócica, es definida como cualquier infección causada por el microorganismo coco grampositivo Streptococcus pneumoniae, también llamado neumococo [1,2]. Las infecciones por este microrganismo representan una de las principales causas de morbimortalidad en todo el mundo $[3,4]$.

La EPN se puede agrupar en dos grandes categorías: invasiva y no invasiva [5]. Esta última es aquella donde la infección se produce en sitios corporales que normalmente no son estériles: sinusitis, otitis media aguda y neumonía no bacteriémica adquirida en la comunidad (NAC) [1] (figura 1).

La S. pneumoniae es responsable de más del $25 \%$ de las neumonías bacterianas, siendo esta infección pulmonar la principal forma de presentación de la EPN no invasiva [1]. La incidencia de NAC alrededor del mundo varía, siendo de alrededor de 1,6-11,6 casos por cada 1.000 personas en Europa [5]; además, en Estados Unidos se estableció que 266,8 casos por cada 100.000 personas con NAC requirieron hospitalización [6]. Sin embargo, estas últimas cifras han disminuido luego de la aplicación de la vacuna contra neumococo, llegando a 54,8 casos por cada 100.000 personas al año $[7,8]$.

Por su parte, la EPN invasiva se define como aquella infección confirmada por aislamiento de neumococo en sitios corporales que son normalmente estériles, como la sangre y el líquido cefalorraquídeo [1]. Siendo así, se reconoce como EPN invasiva la neumonía por neumococo 


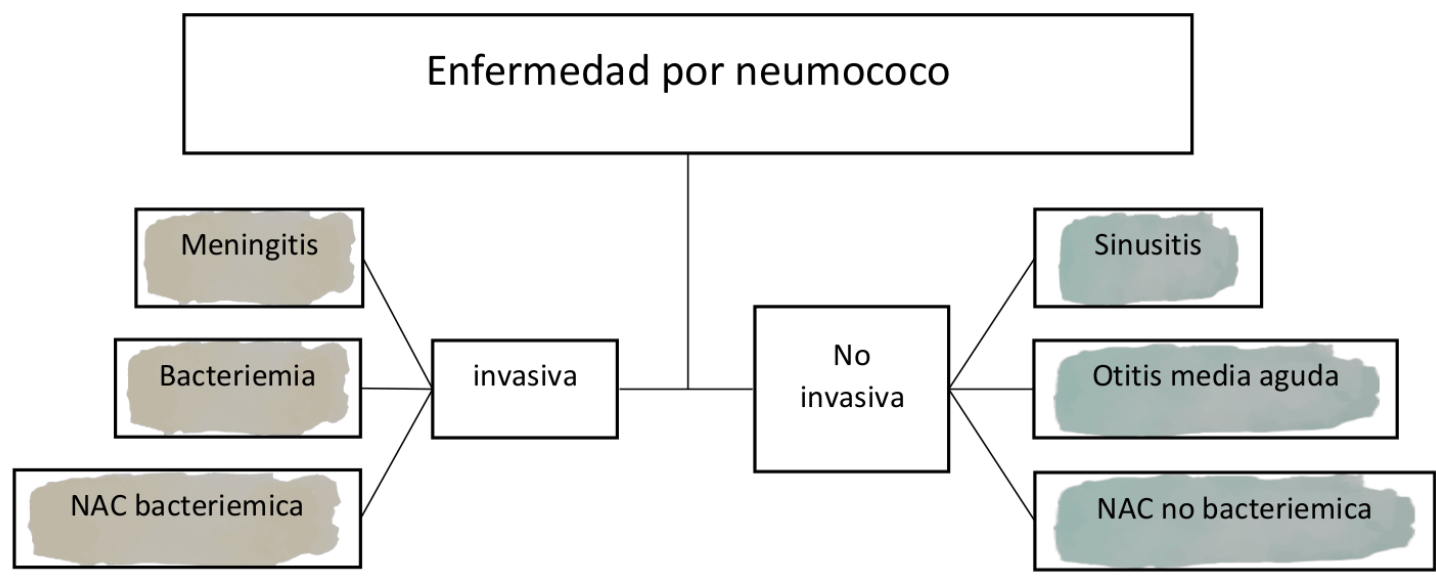

Figura 1: Clasificación de la enfermedad por neumococo

NAC: neumonía adquirida en la comunidad.

Fuente: elaboración con base en Drijkoningen \& Rohde [5].

bacteriémica, la bacteriemia neumocócica sin un foco primario y la meningitis por neumococo (figura 1) [5].

La neumonía se ha descrito como el origen más común de la infección en la EPN invasiva, con cerca del $53 \%$ de los casos; de igual forma la presencia de bacteriemia sin un foco se ha descrito en alrededor del $40 \%$ de los casos y la meningitis en el $5 \%$ [5]. La incidencia de la EPN invasiva se ha mostrado fuertemente relacionada con la edad, presentándose su mayor tasa de incidencia en niños menores de 2 años y en adultos mayores de 65 años [9,10]. Los pacientes inmunocomprometidos tienen un riesgo mucho más elevado de padecer EPN invasiva [1].

Dentro de los principales factores de riesgo para enfermedad neumocócica invasiva (figura 2) se incluyen adultos mayores, pacientes con enfermedades crónicas (enfermedad cardiovascular, enfermedades pulmonares crónicas, enfermedad renal crónica (ERC) y diabetes mellitus no controlada), fumadores, esplenectomizados, inmunosuprimidos y con neoplasias hematológicas malignas [11-16]. Otros factores de riesgo descritos incluyen fistula de líquido cefalorraquídeo y usuarios de implantes cocleares [17-20].

\section{EPN, ERC y diabetes mellitus}

Los pacientes con ERC llevan consigo, como consecuencia de padecer esta enfermedad, una disfunción a nivel de la respuesta inmunológica, tanto innata como adaptativa, lo cual los predispone a desarrollar infecciones [1]; de igual forma, estas personas presentan una retención de compuestos nitrogenados y de citoquinas crónica, sustancias que en situaciones normales son eliminadas por los riñones. Esta condición provoca una disminución de la 


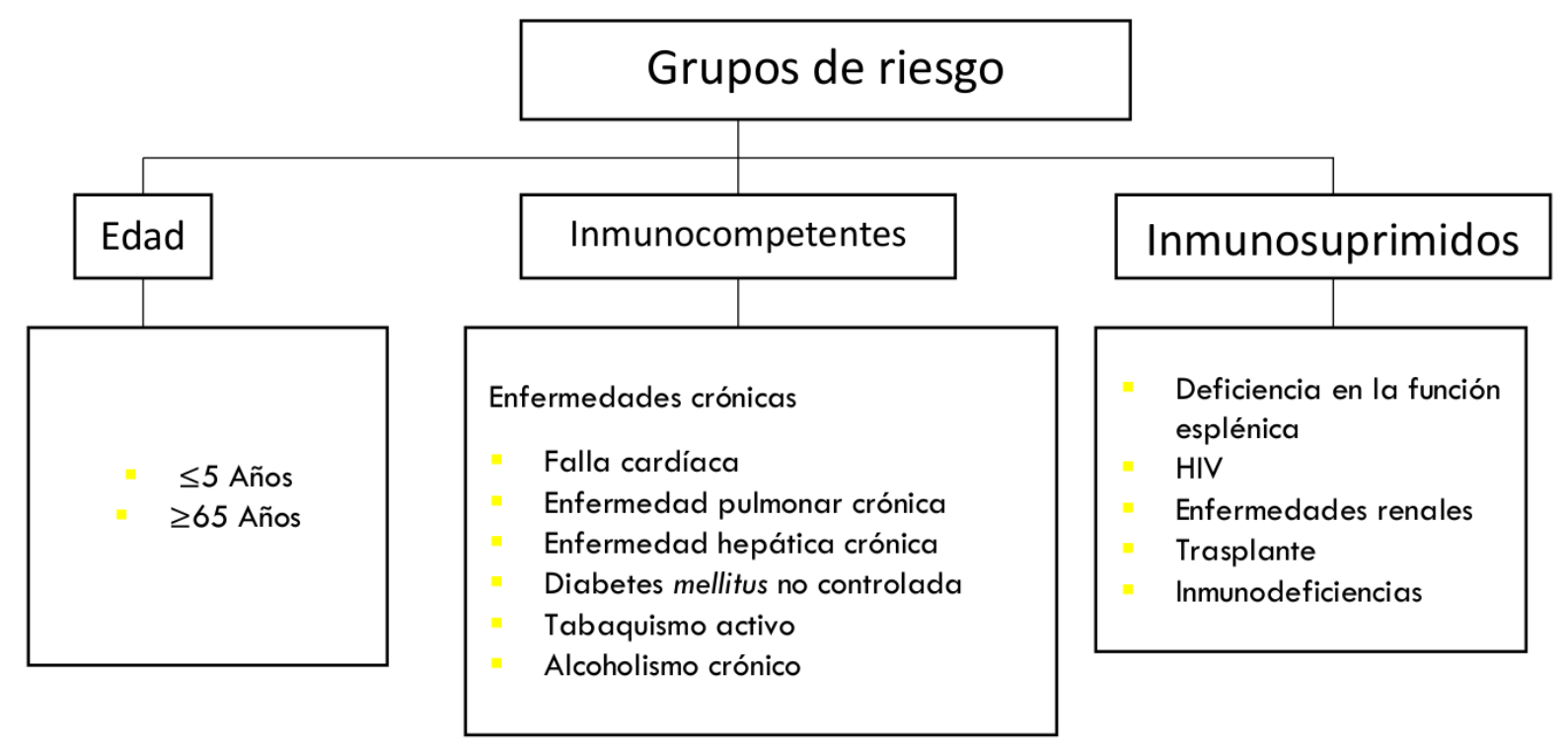

Figura 2: Principales factores de riesgo para enfermedad por neumococo invasiva

Fuente: elaboración propia.

funcionalidad inmunológica que se evidencia en la disminución de la capacidad bactericida de los neutrófilos y los monocitos hiperreactivos, y en una subsecuente diferenciación disminuida en las células dendríticas; en la disminución de la producción de células $\mathrm{T}$ tímicas; en el deterioro de la activación de las respuestas de las células T; en la disminución de los linfocitos B, y en la activación de la apoptosis inducida por células T y B [21]. De hecho, las complicaciones de tipo infeccioso están descritas como la segunda causa principal de hospitalización en los pacientes con ERC, solo después de las complicaciones a nivel cardiovascular [22-25].

El termino enfermedad renal diabética (ERD) denota el daño funcional renal inducido por la diabetes mellitus, siendo esta la principal causa de ERC en la actualidad, pues ocasiona hasta el $40 \%$ de los casos [26].

Cerca del $30 \%$ de los pacientes diabéticos desarrollan ERC en algún momento de su vida [27-31]. Estos pacientes son de alto riesgo debido a que ambas condiciones (la diabetes y la ERC) son factores de riesgo descritos para el desarrollo de enfermedades infecciosas debido a un estado de inmunosupresión [32,33].

La NAC es la segunda causa más común de infección en los pacientes con ERC y diabetes, en especial en quienes la ERC se encuentra en estadios finales; esto se ha asociado con un 
aumento en la mortalidad y un mal pronóstico a largo plazo [34], siendo el neumococo el microorganismo bacteriano más frecuentemente aislado [35].

Slinin et al. [36] reportaron una frecuencia de hospitalización por neumonía de 84,4 hospitalizaciones por cada 1.000 pacientes al año en una muestra de 14.859 pacientes en hemodiálisis. En ese estudio también se estableció que el neumococo fue el responsable de alrededor del $53 \%$ de los casos reportados de neumonía en pacientes en terapia dialítica [36], mientras que Bonnave et al. [11] reportaron que la incidencia de neumonía en pacientes receptores de trasplante renal era de alrededor de 2,62 casos por cada 100 pacientes al año. La tasa de mortalidad de los pacientes en terapia dialítica luego de un episodio de neumonía asciende, encontrándose datos que evidencian tasas hasta 16 veces más altas en comparación a la población general [37,38].

La mortalidad a 6 meses luego de un episodio de neumonía en pacientes que se encuentran en su primer año de terapia dialítica fue de 78,3 por cada 100 pacientes al año en 2001. El riesgo de muerte que tuvieron estos pacientes en comparación a los que estaban en diálisis y que no cursaron con un cuadro de neumonía se mostró mucho más alto, persistiendo elevado hasta 48 meses luego de presentar el evento. Asimismo, los pacientes en terapia dialítica con neumonía presentaron un riesgo aumentado de desarrollo de eventos cardiovasculares en los primeros 6 meses en comparación con aquellos que no tuvieron neumonía [37].

\section{Vacunación antineumocócica en ERC}

La vacunación antineumocócica es una de las medidas de prevención que deben usarse para disminuir el riesgo de presentar una EPN [39,40]. En la actualidad, se cuenta con dos vacunas contra este microorganismo: la 13-valente neumocócica conjugada (PCV13 o Prevenar 13) [41], la cual cuenta con los serotipos 1, 3, 4, 5, 6A, 6B, 7F, 9V, 14, 18C, 19A, 19F y 23F, y la polisacárida capsular neumocócica 23- valente (PPSV23 o Pneumovax 23) [42], que cuenta con los serotipos 1, 2, 3, 4, 5, 6B, 7F, 8, 9N, 9V, 10A, 11A, 12F, 14, 15B, 17F, 18C, 19A, 19F, 20, 22F, 23F y $33 \mathrm{~F}$.

En el año 2011 se publicó un estudio retrospectivo realizado en 118.533 pacientes con ERC que eran manejados con hemodiálisis y en el cual se encontró que la vacunación antineumocócica se asoció con una disminución estadísticamente significativa del riesgo de mortalidad, mortalidad por causas cardiovasculares y hospitalización por bacteriemia, resaltando la asociación pequeña, pero significativa, entre vacunación antineumocócica y disminución en el riesgo de mortalidad [43]. 
Sin embargo, en los pacientes con ERC o en diálisis se ha evidenciado una respuesta disminuida frente a la inmunización contra neumococo. En el año 2009, por ejemplo, se publicó un estudio en el que se buscó evaluar la respuesta de anticuerpos a la vacuna antineumocócica PPSV23 en pacientes con ERC en comparación con pacientes previamente sanos; en dicha investigación se tomaron muestras de sangre antes de la vacunación y cuatro semanas después de la misma, midiéndose anticuerpos específicos contra antígenos neumocócicos completos mediante la técnica ELISA, y se encontró que luego de la vacunación el $21 \%$ de los pacientes con ERC presentó una hiporespuesta a la inmunización y desarrolló más episodios de infecciones por neumococo. A su vez, el aumento de la proporción de los títulos de IgGantineumocócica en el grupo de ERC fue significativamente menor respecto al grupo de pacientes sanos, concluyéndose así que, pese a que la mayoría de los pacientes con ERC respondieron adecuadamente a la vacuna PPSV23, una cantidad sustancial no logró una respuesta de anticuerpos adecuada [44].

Por otro lado, en el año 2016 se publicó un estudio en donde se evaluó la inmunogenicidad de la vacuna PCV13 en pacientes con ERC en estadios terminales y en manejo con terapia dialítica y se encontró una disminución significativa en las concentraciones de anticuerpos para la mayoría de los serotipos de PCV13 a los 12 meses de la vacunación, además la tasa general de respuesta a los serotipos individuales también fue baja en ese mismo plazo [45].

Pese a que la eficacia general de la vacunación contra neumococo en la población con ERC no está del todo definida, la inmunización se recomienda fuertemente debido a la alta mortalidad asociada a EPN invasiva y al bajo riesgo de reacciones adversas ocasionadas por las vacunas [34, 46-48].

La inmunización contra neumococo está recomendada en pacientes con ERC no dependientes de terapia de reemplazo renal, en aquellos que cursen con síndrome nefrótico, en los que requieran inmunosupresión y en los diabéticos [34]. De igual forma, las guías KDIGO (Kidney Disease Improving Global Outcomes) [49] recomendaron la vacunación contra neumococo en todos los pacientes con tasa de filtración glomerular $<30 \mathrm{~mL} / \mathrm{min} / 1.73 \mathrm{~m}^{2}$, tal como se resume en la tabla 1.

Las recomendaciones actuales en cuanto al esquema de inmunización contra neumococo en pacientes con ERC varían según las características del paciente; si ha recibido o no dosis previas de PPSV23 o PCV13, o si, por el contrario, no ha recibido ninguna inmunización [50]. En pacientes menores de 65 años se sugiere un esquema conjugado de PPSV23 más PCV13, mientras que en pacientes mayores a esta edad solo se debe administrar la vacuna polisacárida. 
Tabla 1: Recomendaciones de los diferentes consensos para inmunización antineumocócica en pacientes con enfermedad renal crónica

\begin{tabular}{|l|l|}
\hline \multicolumn{1}{|c|}{ GUIA } & \multicolumn{1}{c|}{ RECOMENDACIÓN } \\
\hline \multirow{2}{*}{ KDIGO-ERC 2012 } & $\begin{array}{l}\text { 1. Se recomienda que en todos los adultos con TFGe }<30 \mathrm{ml} / \mathrm{min} / 1,73 \mathrm{~m}^{2} \text { (Categorías } \\
\text { G4-G5) y aquellas con un riesgo elevado de infección neumocócica ( } \mathrm{p} \text {, ej, Síndrome } \\
\text { nefrótico, diabetes o aquellas que reciben inmunosupresor) reciban la vacuna antine- } \\
\text { umocócica polivalente a menos que esté contraindicada. } \\
\text { 2. Se recomienda que todos los adultos con ERC que hayan recibido vacuna antineu- } \\
\text { mocócica se les ofrezca revacunación dentro de 5 años. }\end{array}$ \\
\hline ACIP 2019 & $\begin{array}{l}\text { 1. No se recomienda usar indiscriminadamente la vacuna PCV13 para todos los adultos } \\
\text { mayores de 65 años. } \\
\text { 2. Se recomienda el uso de la vacuna PCV23. } \\
\text { 3. Se recomienda seleccionar pacientes de riesgo para el uso de PCV13. }\end{array}$ \\
\hline
\end{tabular}

Fuente: elaboración con base en documento de la CKD Evaluation and Management [49].

Para los pacientes que han recibido inmunización previamente con la vacuna PPSV23, las recomendaciones dictan esperar al menos 1 año posterior a esta inmunización para administrar una dosis de la vacuna PCV13 (tabla 2) [50].

Tabla 2: Recomendaciones del Comité Asesor sobre Prácticas de Vacunación para inmunización antineumocócica en pacientes con enfermedad renal crónica

\begin{tabular}{|c|c|c|c|}
\hline \multicolumn{4}{|c|}{ RECOMENDACIONES EN ADULTOS BASADAS EN RIESGO ACIP-CDC } \\
\hline & DOSIS INICIAL & SEGUNDA DOSIS & TERCERA DOSIS \\
\hline $\begin{array}{c}\text { Sujetos que no reciben } \\
\text { PPV23 previa }\end{array}$ & PCV13 & PPV23 en 8 semanas & \\
\hline $\begin{array}{c}\text { Sujetos que recibieron } \\
\text { PPV23 previa }\end{array}$ & PPV23 previa & $\begin{array}{c}\text { PCV13 en 12 meses } \\
\text { o más }\end{array}$ & PPV23 en 8 semanas* \\
\hline
\end{tabular}

*Pacientes inmunosuprimidos: con inmunodeficiencias congénitas o adquiridas, infección por VIH, enfermedad renal crónica o síndrome nefrótico, leucemias, linfomas, tumores diseminados, drogas inmunosupresoras, trasplante de órgano sólido o mieloma múltiple.

Fuente: elaboración con base en documento de la CKD Evaluation and Management [49].

Por su parte, en los pacientes que no hayan recibido inmunización previamente contra neumococo y sean mayores de 19 años al momento recibir el diagnóstico de ERC, se recomienda administrar una dosis de la vacuna PCV13 y esperar un lapso de al menos 8 semanas para aplicar la primera dosis de la vacuna PPSV23, luego de lo cual se debe esperar al menos 5 años para aplicar la segunda dosis de esta última vacuna (Tabla 2) [50]. 
En los pacientes mayores de 65 años se deberá administrar una dosis final de la vacuna PPSV23 al menos 5 años después de la dosis más reciente de PPSV23 [50].

De igual forma, en los pacientes receptores de trasplante renal está recomendada la inmunización con las vacunas actuales (PCV13 y PPSV23) en los esquemas previamente descritos; además, se debe repetir la dosis de la vacuna neumocócica conjugada cuando el paciente cumpla 65 años. Para esta población no solo se debe tener en cuenta la inmunización individual, sino también la de todo su círculo cercano: los trabajadores de la salud que estén en contacto con el paciente, los contactos personales más cercanos e, incluso, las mascotas, todo esto con el fin de reducir el riesgo de transmisión de la enfermedad en el receptor de trasplante [34].

\section{Conclusiones}

La ERC y la diabetes mellitus son factores de riesgo importantes para el desarrollo de enfermedades infecciosas como la enfermedad neumocócica. La vacunación es la medida preventiva más efectiva para evitar complicaciones infecciosas en el mundo, es por esto que los médicos que atienden pacientes con estas dos condiciones deben priorizar la administración de los esquemas de vacunación antineumocócica recomendados.

\section{Contribución de los autores}

Jorge Rico Fontalvo, Rodrigo Daza Arnedo, Nehomar Pájaro Galvis, Victor Leal Martinez, Christian Perez Calvo, Emilio Abuabarak Franco: redacción de documento. Isabella Uparella Gulfo, Yasir Valle Villadiego, Laura Aguilera Rios, Carol Ceballos Diaz: gráficas y tablas. Jesús Díaz Guzmán, Isabel Guete García: busqueda bibliográfica.

\section{Conflicto de intereses}

Ninguno declarado por los autores.

\section{Agradecimientos}

Ninguno declarado por los autores.

\section{Financiación}

Ninguna declarada por los autores. 


\section{Referencias}

[1] Vandecasteele SJ, Ombelet S, Blumental S, Peetermans WE. The ABC of pneumococcal infections and vaccination in patients with chronic kidney disease. Clin Kidney J. 2015;8(3):318-24. https://dx.doi.org/10.1093/ckj/sfv030. 个Ver página 2, 3

[2] Ludwig E, Bonanni P, Rohde G, Sayiner A, Torres A. The remaining challenges of pneumococcal disease in adults. Eur Respir Rev. 2012;21(123):57-65. https://x.doi.org/10.1183/ 09059180.00008911. $\uparrow$ Ver página 2

[3] Aliberti S, Mantero M, Mirsaeidi M, Blasi F. The role of vaccination in preventing pneumococcal disease in adults. Clin Microbiol Infect. 2014;20(Suppl 5):52-8. https://x.doi.org/ 10.1111/1469-0691.12518. $\uparrow$ Ver página 2

[4] Varon E, Mainardi JL, Gutmann L. Streptococcus pneumoniae: still a major pathogen. Clin Microbiol Infect. 2010;16(5):401. https:/dx.doi.org/10.1111/j.1469-0691.2010.03190.x. $\uparrow V e r$ página 2

[5] Drijkoningen JJC, Rohde GGU. Pneumococcal infection in adults: burden of disease. Clin Microbiol Infect. 2014;20(Suppl 5):45-51. https:/dx.doi.org/10.1111/1469-0691.12461. 个Ver página 2,3

[6] Marston BJ, Plouffe JF, File TM, Hackman BA, Salstrom SJ, Lipman HB, et al. Incidence of community-acquired pneumonia requiring hospitalization. Results of a population-based active surveillance Study in Ohio. The Community-Based Pneumonia Incidence Study Group. Arch Intern Med. 1997;157(15):1709-18. ^Ver página 2

[7] Griffin MR, Zhu Y, Moore MR, Whitney CG, Grijalva CG. U.S. Hospitalizations for Pneumonia after a Decade of Pneumococcal Vaccination. N Engl J Med. 2013;369(2):155-63.

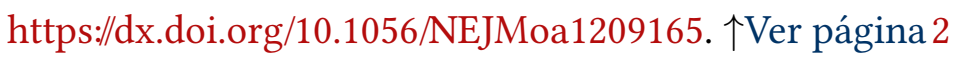

[8] Mackenzie GA, Hill PC, Jeffries DJ, Hossain I, Uchendu U, Ameh D, et al. Effect of the introduction of pneumococcal conjugate vaccination on invasive pneumococcal disease in The Gambia: a population-based surveillance study. Lancet Infect Dis. 2016;16(6):703-11. https://x.doi.org/10.1016/s1473-3099(16)00054-2. 个Ver página 2

[9] Robinson KA, Baughman W, Rothrock G, Barrett NL, Pass M, Lexau C, et al. Epidemiology of invasive Streptococcus pneumoniae infections in the United States, 1995-1998: Opportunities for prevention in the conjugate vaccine era. JAMA. 2001;285(13):1729-35.

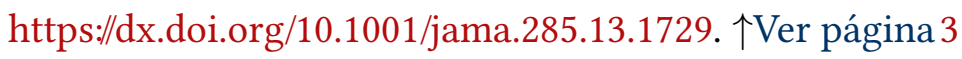


[10] Marston BJ, Plouffe JF, File TM, Hackman BA, Salstrom SJ, Lipman HB, et al. Incidence of Community-Acquired Pneumonia Requiring Hospitalization. Results of a Population-Based Active Surveillance Study in Ohio. Arch Intern Med. 1997;157(15):1709-18. https://x.doi.org/ 10.1001/archinte.1997.00440360129015. 个Ver página 3

[11] Bonnave C, Mertens D, Peetermans W, Cobbaert K, Ghesquiere B, Deschodt M, et al. Adult vaccination for pneumococcal disease: a comparison of the national guidelines in Europe. Eur J Clin Microbiol Infect Dis. 2019;38(4):785-91. https:/dx.doi.org/10.1007/

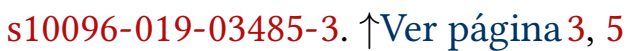

[12] Cruickshank HC, Jefferies JM, Clarke SC. Lifestyle risk factors for invasive pneumococcal disease: a systematic review. BMJ Open. 2014;4(6):e005224. https://dx.doi.org/10.1136/ bmjopen-2014-005224. $\uparrow$ Ver página 3

[13] Torres A, Blasi F, Dartois N, Akova M. Which individuals are at increased risk of pneumococcal disease and why? Impact of COPD, asthma, smoking, diabetes, and/or chronic heart disease on community-acquired pneumonia and invasive pneumococcal disease: Thorax.

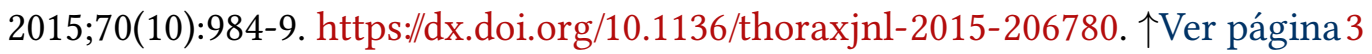

[14] Mor A, Thomsen RW, Ulrichsen SP, Sørensen HT. Chronic heart failure and risk of hospitalization with pneumonia: A population-based study. Eur J Intern Med. 2013;24(4):349-53. https:/dx.doi.org/10.1016/j.ejim.2013.02.013. $\uparrow$ Ver página 3

[15] Shea KM, Edelsberg J, Weycker D, Farkouh RA, Strutton DR, Pelton SI. Rates of Pneumococcal Disease in Adults With Chronic Medical Conditions. Open Forum Infect Dis. 2014;1(1):ofu024. https://dx.doi.org/10.1093/ofid/ofu024. 个Ver página 3

[16] Juthani-Mehta M, De Rekeneire N, Allore H, Chen S, O’Leary JR, Bauer DC, et al. Modifiable Risk Factors for Pneumonia Requiring Hospitalization of Community-Dwelling Older Adults: The Health, Aging, and Body Composition Study. J Am Geriatr Soc. 2013;61(7):11118. https:/dx.doi.org/10.1111/jgs.12325. 个Ver página 3

[17] Ochoa-Gondar O, Vila-Corcoles A, de Diego C, Arija V, Maxenchs M, Grive M, et al. The burden of community-acquired pneumonia in the elderly: the Spanish EVAN-65 study. BMC Public Health. 2008;8:222. https://dx.doi.org/10.1186/1471-2458-8-222. 个Ver página 3

[18] Jaume A, Salle F, Devita A, Martínez F, Sgarbi N. Fístula de líquido cefalorraquídeo postraumática: propuesta de algoritmo diagnóstico y terapéutico. Arch Med Int. 2015;37(1):4752. $\uparrow$ Ver página 3

[19] Gil-Prieto R, Garcia-Garcia L, Alvaro-Meca A, Mendez C, Garcia A, de Miguel AG. The burden of hospitalisations for community-acquired pneumonia (CAP) and pneumococcal 
pneumonia in adults in Spain (2003-2007). Vaccine. 2011;29(3):412-6. https:/dx.doi.org/10. 1016/j.vaccine.2010.11.025. 个Ver página 3

[20] Chidiac C. Pneumococcal infections and adult with risk factors. Med Mal Infect. 2012;42(10):517-24. https://dx.doi.org/10.1016/j.medmal.2012.04.003. 个Ver página 3

[21] Syed-Ahmed M, Narayanan M. Immune Dysfunction and Risk of Infection in Chronic Kidney Disease. Adv Chronic Kidney Dis. 2019;26(1):8-15. https:/dx.doi.org/10.1053/j.ackd. 2019.01.004. $\uparrow$ Ver página 4

[22] Ishigami J, Matsushita K. Clinical epidemiology of infectious disease among patients with chronic kidney disease. Clin Exp Nephrol. 2019;23(4):437-47. https://x.doi.org/10.1007/ s10157-018-1641-8. 个Ver página 4

[23] Saran R, Robinson B, Abbott KC, Agodoa LYC, Bhave N, Bragg-Gresham J, et al. US Renal Data System 2017 Annual Data Report: Epidemiology of Kidney Disease in the United States. Am J Kidney Dis. 2018;71(3 Suppl 1):A7. https://dx.doi.org/10.1053/j.ajkd.2018.01.002. 个Ver página 4

[24] James MT, Laupland KB, Tonelli M, Manns BJ, Culleton BF, Hemmelgarn BR. Risk of Bloodstream Infection in Patients With Chronic Kidney Disease Not Treated With Dialysis. Arch Intern Med. 2088;168(21):2333-9. https:/dx.doi.org/10.1001/archinte.168.21.2333. $\uparrow$ Ver página 4

[25] Dalrymple LS, Katz R, Kestenbaum B, de Boer IH, Fried L, Sarnak MJ, et al. The Risk of Infection-Related Hospitalization With Decreased Kidney Function. Am J Kidney Dis.

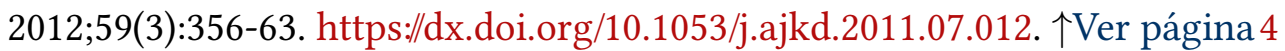

[26] Alicic RZ, Rooney MT, Tuttle KR. Diabetic Kidney Disease: Challenges, Progress, and Possibilities. Clin J Am Soc Nephrol. 2017;12(12):2032-45. https://dx.doi.org/10.2215/CJN. 11491116. $\uparrow$ Ver página 4

[27] Tesch GH. Diabetic nephropathy - is this an immune disorder? Clin Sci (Lond).

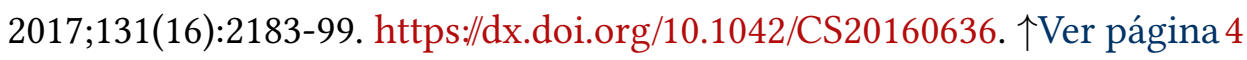

[28] Kidney Disease: Improving Global Outcomes (KDIGO) Diabetes Work Group. KDIGO 2020 Clinical Practice Guideline for Diabetes Management in Chronic Kidney Disease. Kid-

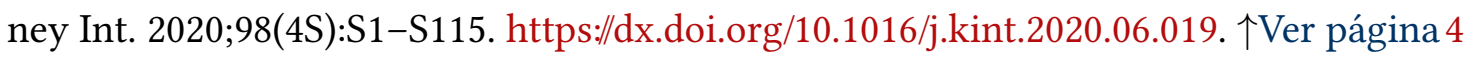

[29] Gross JL, de Azevedo MJ, Silveiro SP, Canani LH, Caramori ML, Zelmanovitz T. Diabetic Nephropathy: Diagnosis, Prevention, and Treatment. Diabetes Care. 2005;28(1):164-76. https://dx.doi.org/10.2337/diacare.28.1.164. $\uparrow$ Ver página 4 
[30] Lim A. Diabetic nephropathy - complications and treatment. Int J Nephrol Renovasc Dis.

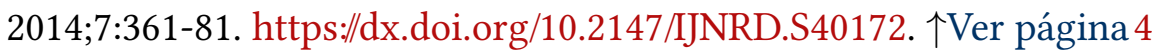

[31] Umanath K, Lewis JB. Update on Diabetic Nephropathy: Core Curriculum 2018. Am J Kidney Dis. 2018;71(6):884-95. https://x.doi.org/10.1053/j.ajkd.2017.10.026. $\uparrow$ Ver página 4

[32] Casqueiro J, Casqueiro J, Alves C. Infections in patients with diabetes mellitus: A review of pathogenesis. Indian J Endocr Metab. 2012;16(Suppl 1):s27-36. https://dx.doi.org/10.4103/ 2230-8210.94253. $\uparrow$ Ver página 4

[33] Pahl MV, Vaziri ND. Immune Function in Chronic Kidney Disease. En: Chronic Renal Disease. Elsevier; 2015 [citado agosto 9 de 2020]. p. 285-97. Disponible en: https:/linkinghub. elsevier.com/retrieve/pii/B978012411602300024X. $\uparrow$ Ver página 4

[34] Krueger KM, Ison MG, Ghossein C. Practical Guide to Vaccination in All Stages of CKD, Including Patients Treated by Dialysis or Kidney Transplantation.Am J Kidney Dis. 2020;75(3):417-25. https://dx.doi.org/10.1053/j.ajkd.2019.06.014. $\uparrow$ Ver página 5, 6, 8

[35] Viasus D, Garcia-Vidal C, Cruzado JM, Adamuz J, Verdaguer R, Manresa F, et al. Epidemiology, clinical features and outcomes of pneumonia in patients with chronic kidney disease. Nephrol Dial Transplant. 2011;26(9):2899-906. https://dx.doi.org/10.1093/ndt/gfq798. 个Ver página 5

[36] Slinin Y, Foley RN, Collins AJ. Clinical epidemiology of pneumonia in hemodialysis patients: the USRDS waves 1, 3, and 4 study. Kidney Int. 2006;70(6):1135-41. https:/dx.doi.org/ 10.1038/sj.ki.5001714. $\uparrow$ Ver página 5

[37] Dinits-Pensy M, Forrest GN, Cross AS, Hise MK. The Use of Vaccines in Adult Patients With Renal Disease. Am J Kidney Dis. 2005;46(6):997-1011. https://dx.doi.org/10.1053/j.ajkd. 2005.08.032. $\uparrow$ Ver página 5

[38] Sarnak MJ, Jaber BL. Pulmonary infectious mortality among patients with end-stage renal

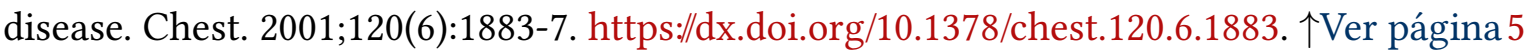

[39] Portolés-Pérez J, Marqués-Vidas M, Marques-Vidas M, Picazo JJ, González- Romo F, García-Rojas A, et al. Recomendaciones de vacunación frente a neumococo en enfermos renales en España. Nefrología. 2014;34(5):545-692. https:/doi.org/10.3265/Nefrologia.pre2014. May.12534. $\uparrow$ Ver página 5

[40] Ficha tecnica Prevenar 13 suspención inyectable. European Medicines Agency. Science Medicines Health; [citado julio 15 de 2013]. Disponible en: http:/www.ema.europa.eu/docs/ es_ES/document_library/EPAR-Product_Information/human/001104/WC500057247.pdf. 个Ver página 5 
[41] Jefferies JMC, Macdonald E, Faust SN, Clarke SC. 13-valent pneumococcal conjugate vaccine (PCV13). Hum Vaccin. 2011;7(10):1012-8. https://dx.doi.org/10.4161/hv.7.10.16794. $\uparrow$ Ver página 5

[42] Daniels CC, Rogers PD, Shelton CM. A Review of Pneumococcal Vaccines: Current Polysaccharide Vaccine Recommendations and Future Protein Antigens. J Pediatr Pharmacol Ther. 2016;21(1):27-35. https://dx.doi.org/10.5863/1551-6776-21.1.27. 个Ver página 5

[43] Gilbertson DT, Guo H, Arneson TJ, Collins AJ. The association of pneumococcal vaccination with hospitalization and mortality in hemodialysis patients. Nephrol Dial Transplant. 2011;26(9):2934-9. https://dx.doi.org/10.1093/ndt/gfq853. 个Ver página 5

[44] Mahmoodi M, Aghamohammadi A, Rezaei N, Lessan-Pezeshki M, Pourmand G, Mohagheghi MA, et al. Antibody response to pneumococcal capsular polysaccharide vaccination in patients with chronic kidney disease. Eur Cytokine Netw. 2009;20(2):69-74. https:

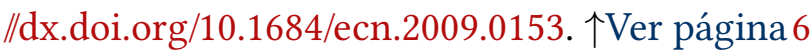

[45] Mitra S, Stein GE, Bhupalam S, Havlichek DH. Immunogenicity of 13-Valent Conjugate Pneumococcal Vaccine in Patients 50 Years and Older with End- Stage Renal Disease and on Dialysis. Clin Vaccine Immunol. 2016;23(11):884- 7. https:/dx.doi.org/10.1128/CVI.00153-16. $\uparrow$ Ver página 6

[46] Kato S, Chmielewski M, Honda H, Pecoits-Filho R, Matsuo S, Yuzawa Y, et al. Aspects of Immune Dysfunction in End-stage Renal Disease. Clin J Am Soc Nephrol. 2008;3(5):1526-33. https:/dx.doi.org/10.2215/cjn.00950208. $\uparrow$ Ver página 6

[47] DaRoza G, Loewen A, Djurdjev O, Love J, Kempston C, Burnett S, et al. Stage of chronic kidney disease predicts seroconversion after hepatitis B immunization: earlier is better. Am J Kidney Dis. 2003;42(6):1184-92. https:/dx.doi.org/10.1053/j.ajkd.2003.08.019. 个Ver página 6

[48] Shen AK, Kelman JA, Warnock R, Zhang W, Brereton S, McKean S, et al. Beneficiary characteristics and vaccinations in the end-stage renal disease Medicare beneficiary population, an analysis of claims data 2006-2015. Vaccine, 2017;35(52):7302-8. https:/dx.doi.org/ 10.1016/j.vaccine.2017.10.105. $\uparrow$ Ver página 6

[49] CKD Evaluation and Management. KDIGO; 2012 [citado agosto 1 de 2020]. Disponible en: https:/kdigo.org/guidelines/ckd-evaluation-and-management/. $\uparrow$ Ver página 6, 7

[50] Reddy S, Chitturi C, Yee J. Vaccination in Chronic Kidney Disease. Adv Chronic Kidney

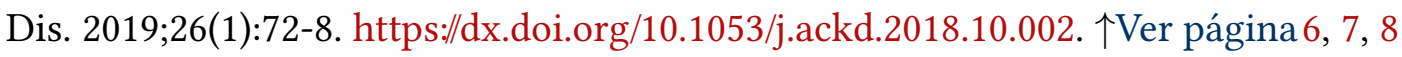

